Voix et Images

\title{
La thématique de la forêt dans deux romans ontarois
}

\section{Yolande Grisé}

Volume 14, numéro 2 (41), hiver 1989

L’édition littéraire au Québec

URI : https://id.erudit.org/iderudit/200774ar

DOI : https://doi.org/10.7202/200774ar

Aller au sommaire du numéro

\section{Éditeur(s)}

Université du Québec à Montréal

\section{ISSN}

0318-9201 (imprimé)

1705-933X (numérique)

Découvrir la revue

\section{Citer cet article}

Grisé, Y. (1989). La thématique de la forêt dans deux romans ontarois. Voix et Images, 14(2), 269-280. https://doi.org/10.7202/200774ar d'utilisation que vous pouvez consulter en ligne.

https://apropos.erudit.org/fr/usagers/politique-dutilisation/ 


\section{La thématique de la forêt dans deux romans ontarois*}

\section{par Yolande Grisé, Université d'Ottawa}

On sait depuis longtemps que les histoires racontées par les écrivains ne sont, en fait, que prétextes pour exprimer des images. D'autre part, on observe dans la littérature occidentale que la forêt est l'image par excellence de la puissance sacrée de la vie. Mais la vie n'est-elle pas fille de la mort ${ }^{1}$ ? Ainsi trouvet-on aux sources mêmes de cette littérature occidentale que constituent les contes populaires, l'image de la forêt, qui en forme l'un des attributs caractéristiques et permanents. Or, dans son ouvrage sur les Racines historiques du conte merveilleux, Vladimir Propp a démontré le lien étroit qui existe entre la forêt du conte et celle figurant dans les rites d'initiation du monde entier, à ce point que là où la forêt n' existe pas, les broussailles en tiennent lieu. ${ }^{2}$

Cette présence obligatoire de la forêt observée dans les rites archaïques s'explique par le rôle fonctionnel qu'elle occupe dans les rites de passage, lesquels expriment et formalisent la mutation profonde nécessaire à la traversée des différentes étapes de la vie. Cette mort momentanée du "vieil homme» indispensable à toute renaissance de «l'homme nouveau» que consacre le rite initiatique, ne pouvait avoir lieu que dans un emplacement retiré, infranchissable, en rapport direct avec le monde des morts: la forêt des récits traditionnels reflète le souvenir de la forêt initiatique en tant qu'entrée du royaume des morts.

En parcourant les récits de l'Ontario français ${ }^{3}$, on remarque la présence significative de la forêt, en particulier dans les Engagés du Grand Portage, le

* Ce texte est une version remaniée d'une communication présentée au colloque international tenu à l'automne 1986 sur l'Homme et la forêt et organisé par le Centre d'études canadiennes de l'Université de Dijon.

1 Gaston Bachelard, la Terre et les rêveries de la volonté, Paris, Librairie José Corti, 1948 , p. 251.

2 Vladimir Propp, les Racines historiques du conte merveilleux, traduit du russe par Lise Gruel-Apart, Paris, Gallimard, 1983, p. 69 (Bibliothèque des sciences humaines).

3 L'étude de la littérature de l'Ontario français est relativement récente. En effet, c'est en 1977 que le Département des lettres françaises de l'Université d'Ottawa acceptait de créer un cours sur la Littérature outaouaise et franco-ontarienne en vue d'explorer ce nouveau champ de recherche dans le domaine des lettres canadicnnesfrançaises. Pour une vue d'ensemble du corpus littéraire ontarois, on voudra bien se reporter à l'ouvrage de Paul Gay, la Vitalité de l'Ontario français, premier panorama, Ottawa, Éditions du Vermillon, 1986, 240 p. (Padagogus, no 1). 
Flambeau sacré, la Vallée des blés d'or, le Loup de Lafontaine, le Coffre, la Vengeance de l'orignal ou encore le Trappeur du Kabi ${ }^{4}$. Bien qu'imposante, la forêt apparaît le plus souvent dans ces textes comme un simple milieu de vie ou encore comme une fresque impressionnante, objet de répulsion ou de fascination.

En revanche, le traitement du thème de la forêt varie profondément dans deux autres romans: François Duvalet ${ }^{5}$ et la Quête d'Alexandre ${ }^{6}$. Dans ces récits, où chaque auteur raconte à sa manière le parcours initiatique d'un héros qui va, pour ainsi dire, frapper aux portes de la mort avant d'entreprendre une nouvelle vie, la forêt n'apparaît plus seulement comme un espace objectif où le héros doit affronter une série d'épreuves, mais constitue, en tant que médiatrice du monde des puissances obscures, l'épreuve par excellence indispensable à la réalisation du héros. Ici, c'est la forêt septentrionale qui aliène, morcelle et absorbe l'homme tout entier pour enfin le rendre à la vie, transformé. Alchimie de la forêt profonde, foyer occulte de la connaissance qui révèle l'homme à luimême.

Autre fait qui ne manque pas de retenir l'attention dans les deux œuvres choisies, au terme du recensement des principaux éléments qui révèlent cette fonction initiatrice de la forêt: c'est l'image commune qui s'en dégage en dépit de la disparité des époques et des origines culturelles des auteurs. François Duvalet, dont l'intrigue se déroule dans le nord-ouest de l'Ontario, plus précisément dans la région de Chapleau, à l'époque de la crise économique de 1929, prend sa source dans l'expérience et les souvenirs de son auteur, Maurice de Goumois. Né en 1896 à Colmar, en Alsace, il mourut à Montréal en 19707. François Duvalet, paru à Québec en 1954, était son deuxième roman. Par ailleurs, la Quête d'Alexandre, qui raconte l'aventure d'un jeune séminariste québécois à la recherche de son frère disparu dans le nord-est ontarien au début du siècle, s'inspire largement de l'histoire orale des pionniers canadiensfrançais attirés dans ces régions par la perspective d'un sort meilleur ${ }^{8}$. Née en 1923 à Saint-Léon-de-Val-Racine, dans les cantons de l'Est, l'auteure, Hélène Brodeur, a quitté le Québec à l'âge de quatre ans, quand sa famille vint s'établir

4 Léo-Paul Desrosiers, les Engagés du Grand Portage, Paris, Gallimard, 1938; 2e éd. Montréal, Fides, 1946 (Collection du Nénuphar); autres éditions plus récentes. Mariline, le Flambeau sacré, Montréal, Bernard Valiquette, 1944;2e éd., Sudbury, Prise de Parole, 1982. Albertine Hallé, la Vallée des blés d'or, Montréal, éd. F. Pilon, 1948; $2^{e}$ éd. Sudbury, Prise de Parole, 1983. Thomas Marchildon, le Loup de Lafontaine, Sudbury, la Société historique du Nouvel-Ontario, 1955. Jocelyne Villeneuve, le Coffre. Contes, Sudbury, Prise de Parole, 1979. Doric Germain, la Vengeance de l'orignal, Sudbury, Prise de Parole, 1980. Doric Germain, le Trappeur du Kabi, Sudbury, Prise de Parole, 1981.

5 Maurice de Goumois, François Duvalet, Québec, Institut littéraire du Québec, 1954.

6 Hélène Brodeur, Chroniques du Nouvel-Ontario. La Quête d'Alexandre, Montréal, Quinze, 1981 (Prose entière); 2c éd. Sudbury, Prise de Parole, 1986.

7 Yolande Grisé, «Un "Frenchy" à Chapleau au temps de la crise», le Droit, 19 avril 1980, p. 18.

8 Hélène Brodeur, «Faire revivre le passé», Liaison, no 30, printemps 1984, p. 47. 
à Val-Gagné, près de Timmins, dans cette région du nord de l'Ontario communément appelée le Nouvel-Ontario. Depuis les années 60 , elle vit principalement dans la région d'Ottawa. Publié en 1981, la Quête d'Alexandre était son premier roman?.

Il convient d'examiner en premier lieu l'ouvrage d'Hélène Brodeur, plus près de nous par le temps de l'écriture, mais plus éloigné par le temps du récit que celui de Maurice de Goumois, afin de déceler à travers l'image de la forêt qu'il projette l'antique fonction sacrée attribuée à ce lieu dans les rites de passage.

Au moment où le jeune séminariste Alexandre part à la découverte de luimême sur les traces de son frère disparu, c'est l'époque de la Première Guerre mondiale. Notons que le seul rapport établi dans le récit avec ce tragique épisode du début du siècle est l'évocation de la mort toute-puissante (p. 271). De ce côté-ci du monde, dans le nord ontarien, c'est surtout l'époque où des milliers de gens de toutes origines vont, par trains entiers, tenter fortune dans la ruée sauvage qu'entraîne la prospection minière à travers les vastes territoires qui longent la frontière occidentale du Québec, de North Bay à Cochrane en direction sud-nord et qu'on appelle le Nouvel-Ontario.

À la plupart des nouveaux arrivants remplis de rêves et d'espoirs, la terre promise réserve, cependant, de nombreux désenchantements, dont la découverte d'un milieu dit naturel qui s'avère, en fait, totalement étranger. En effet, au premier abord, l'étendue et la sauvagerie des lieux subjuguent l'imagination, réduisent paradoxalement les visions et acculent à l'insignifiance toute entreprise humaine. C'est ainsi qu'à peine entrevue, la forêt illimitée (p. 53) du nord provoque un sentiment d'impuissance qui freine le moindre élan vital. Tout occupé par l'autre (la forêt), l'espace infini écrase, étouffe, anéantit; c'est un lieu investi où rien n'est familier, où l'être découvre subitement le sentiment du néant:

oppressé par cette immensité, fétu de paille emporté à toute vitesse dans ce paysage illimité vers une destination inconnue [Alexandre Sellier] se remémora les paysages plus amènes de son enfance, dans les monts des Cantons de l'Est [...]. (p. 39)

Dans cet espace sans frontières qui défile interminablement, le híros aperçoit ici et là des fermes et des champs qui apparaissent bien minuscules (p. 53). L'angoisse ressentie devant ces étendues occupées; mais désertes, confine à l'aliénation.

9 La trilogie des Chroniques du Nouvel-Ontario est composée également de Entre l'aube et le jour (volume II) et les Routes incertaines (volume III). Hélène Brodeur a reçu trois prix littéraires: le prix Champlain 1981, le prix du NouvelOntario 1984 et le prix le Droit 1985. 
De son côté, Rose Brent, jeune orpheline britannique fraichement immigrée dans le Nouvel-Ontario afin de rejoindre un frère unique qui vient de se marier, voit à son tour se modifier, au fur et à mesure qu'elle pénètre au cou du territoire, dans le train qui s'enfonce sans relâche dans la forêt sombre (p. 142), le tableau idyllique qu'elle se plaisait à imaginer dans son ignorance (p. 140). Dès les premiers regards dans le foisonnement de la forêt, c'est le bris du rêve, la désillusion complète, la brutale révélation de l'impénétrabilité de la nature et l'expérience immédiate d'un très fort sentiment de rejet:

Depuis son départ de Halifax, elle se voyait emportée à toute vitesse dans ce paysage inhumain et apparemment sans limites où les hauts conifères semblaient s'avancer en rangs serrés pour repousser l'intrus qui oserait vouloir y pénétrer. [...] [T]out semblait étranger. (p. 141)

Cet espace incommensurable (p. 39, 140), réducteur et hostile, sécrète comme une sourde menace, une végétation certes abondante, mais austère, rude, voire rébarbative (p. 109). Ainsi, Alexandre découvre sous le soleil une végétation sombre (p. 38), bien différente de celle des forêts d'érable et de merisier de ses montagnes natales (p. 74). Ce sont surtout des conifêres à la taille élevée et aux teintes obscures (p. 53, 57, 141, 142): hautes épinettes (p. 38-39), grands pins gris (p. 57), hautes futaies de trembles vert argenté (p. 39), que viennent éclairer, par-ci par-là, des bouquets de bouleaux blancs (p. 39). Dans cette nature désolée et accidentée, fermente, en des replis humides bâillant à ciel ouvert, l'univers putréfié des muskegs, sorte de marais où il ne pousse que des broussailles et des épinettes rabougries (p. 148).

Les changements de saisons ne viennent guère modifier ce tableau lugubre et menaçant. L'été étale un paysage monotone et sans charme (p. 39) d'où émergent les têtes acérées des épinettes (p. 143) et où se distinguent la surface rugueuse des framboisiers (p. 212), les bouquets durs vert foncé des arbustes de bleuets (p. 53), les fourrés si combustibles (p. 54), la cotte de lichens et de plantes ligneuses et sèches (p. 67) qui recouvre par endroits le sol piqué de fleurs à feu d̀ corolles magenta qui courent dans les baisseurs comme des flammes (p. 53). D'autre part, l'hiver (p. 167) tient en réserve un paysage d'où la neige et le ciel gris drainent toute couleur:

L'étable était un cube gris fer; les arbres où s'accrochaient les dernières feuilles, les conifères étaient noirs; le pont gris enjambait la rivière, muée en sombre serpent dont les anneaux enserraient la lagune de terre où se dressait la maison. L'hiver avait repris son empire sur ce pays austère au paysage monochrome. (p. 184)

Le sentiment d'oppression provoqué par le paysage se répercute jusque dans le monde minéral qui s'accorde à une nature primordiale, farouche, rebutante et piégée:

Le ciel était gris. D'énormes rochers gris et noirs, mouillés par la pluie qui tombait depuis North Bay luisaient sinistrement parmi les arbres comme des bêtes tapies. Les nombreux lacs mêlaient le gris 
sale du ciel aux teintes sombres de leurs eaux. Enfin, dans l'aprèsmidi, on arriva à une clairière dans le bois sombre. (p. 142)

L'hostilité de cette forêt s'exprime, enfin, dans l'horizon déchiqueté qu'étire à l'infini la ligne des arbres. Car ce qui retient l'attention du voyageur dans la masse uniforme de ces bois nordiques, c'est moins le nombre des arbres que le sauvage éventrement qui les traverse et préfigure en quelque sorte le démembrement et la dévastation intérieurs du héros. En effet, deux traits essentiels caractérisent la forêt littéraire d'Hélène Brodeur: les troncs amputés et les troncs foudroyés. C'est ainsi que, sous les yeux du voyageur qui traverse ces contrées, le rempart ordonné des hauts sapins s'effondre par endroits sous l'amoncellement des grands arbres abattus, des tas de branches (p. 272) et des monceaux de broussailles déracinées (p. 53) qui traînent de chaque côté du chemin de fer, sans compter les trouées inattendues qui taillent le rideau résineux en clairières parsemées de souches (p. 57):

Ce nuage de poussière qui brillait au soleil et surtout les souches qu'on voyait partout, voilà ce qui avait le plus frappé Alexandre. Depuis qu'il avait quitté North Bay, il avait pu suivre le passage de l'homme dans cette forêt septentrionale par ces moignons de troncs mutilés qui se multipliaient entre les cabanes autour des exploitations minières, le long des chemins, sur le bord des cours d'eau. (p. 41)

Et encore :

Dans les minuscules clairières des fermiers, dans les vastes superficies des exploitations forestières, toujours les éternelles souches comme des poils de barbe sur une joue mal rasée. (p. 53)

Au tablcau ravagé de la forêt abattue, éventrée, réduite à certains endroits à l'état de taillis, vient se juxtaposer le spectacle tourmenté des torches noires qui émergent abruptement entre les vagues sombres des coniferes:

Les grands arbres noircis dressaient toujours leurs troncs lépreux, tendaient leurs branches décharnées d'où pendaient des lambeaux d'écorce calcinés. [....] Ça et là, des espaces gris, des creux remplis de cendres rappelaient la grande conflagration qui avait balayé la région. (p. 53)

Parcil cataclysme laisse derrière lui une sombre et morne plaine s'étendant à perte de vue et se confondant à l'horizon, hérissée de troncs à demi calcinés (p. 274).

Dans les rites d'initiation, il est attesté que des néophytes subissaient l'ćprcuve du feu, et cela sous les formes les plus variées ${ }^{10}$. Ces brûlures réelles, symboliques par la suite, devaient d'abord purifier la victime de ses points faibles, lui forger une âme nouvelle pour remplir ses nouvelles fonctions et lui êtres bénéfiques pour l'avenir. On voit donc chez Hélène Brodeur la forêt

10 Vladimir Propp, op. cit., p. 125. 
ontarienne aux essences résineuses se dresser comme un énorme bûcher offert à l'étincelle du sacrifice (p. 53). On peut y apercevoir, en préfiguration de la mise à mort indispensable à l'initiation, des troncs mutilés d'où pendaient des lambeaux d'écorce noircie comme la peau des suppliciés (p. 273). Le feu absorbe sa proie tout entière: c'est un véritable fauve (p. 269), une sorte de bête frustrée (p. 272), monstre (p. 269), dévorant (p. 278), hydre aux mille têtes (p. 272) qui couve dans les tourbières de ce rude pays (p. 13).

En réalité, l'initiation des élus constituait une véritable descente aux enfers, dans les entrailles chaudes de la terre où le germe doit se décomposer dans la pourriture afin que naissent l'arbre et l'homme renouvelé. Ainsi, en toutes saisons, la fumée imprègne l'atmosphère de la forêt nord-ontarienne. L'été, les abattis brûlent à foison (p. 53, 146, 278); on apprend à fumer la pipe pour se défendre des moustiques de toutes sortes qui envahissent ces bois traversés de cours d'eau (p. 61); on cuisine à l'extérieur. L'hiver, l'odeur du bois brûlé s'échappe par les cheminées des poêles qui craquent (p. 194); on voit la fumée des lampes à l'huile qu'on craint de renverser (p. 189). Et, en tout temps, la fumée âcre, noire et huileuse (p. 39) des locomotives, machines infernales bourrées de charbon, zèbre le ciel de ses traînées de suie (p. 142). Pareille odeur de soufre n'est pas loin d'évoquer l'antre de quelque lieu infernal.

En effet, dans cette forêt surchauffée par les températures élevées de certains étés (p. 278) - [Alexandre] n'aurait jamais cru qu'il pût faire aussi chaud dans ces territoires septentrionaux (p. 38) -, on est aux portes mêmes de l'enfer, qui s'abattent avec fracas quand l'incendie éclate: avec un bruit d'enfer, l'incendie se rapprochait toujours (p. 272). Et c'est la catastrophe d'une envergure inimaginable, qui transforme la forêt en gigantesque fournaise:

L'incendie fonçait sur eux avec un grondement de cent locomotives lancées à toute vitesse, des locomotives qui auraient circulé sur un pont. Le vent hurlait et précipitait sur eux des étincelles et des braises. [...] Un à un, les grands conifères flambaient comme des torches. Puis la chaleur les faisait exploser avec des bruits d'obus tandis que les débris enflammés, portés par le vent, pleuvaient à cent pieds en avant, allumant de nouveaux foyers d' incendie [...] Déjà les grands arbres de leur côté flambaient, au pied même de la colline où ils s'étaient réfugiés. Une détonation retentit toute proche et une tête d'épinette enflammée tomba carrément sur leur groupe. (p. 269)

Pour se mettre à l'abri, le héros et les siens n'ont-ils pas choisi de creuser des terricrs dans le sol d'un ancien cimetière indien et de s'y allonger comme des morts recouverts du linceul de lcurs couvertures mouillées? Ici, le mimétisme de la mort est patent (p. 269).

L'épreuve du feu confêre à l'initié une âme nouvelle marquée de son expćrience de l'au-delà, où il a communié à la nature divine et immortelle qui, scule, pcut lui permettre d'acquérir les qualités essentielles à sa nouvelle vie. Dans le récit, le point ultime de l'initiation héroïque est atteint au paroxysme du 
sacrifice, au moment où, dans ce décor dantesque, la forêt se tord dans un grand tourbillon de flammes, de vapeurs empoisonnées et d'explosions. À l'effroi ressenti devant un sinistre aussi redoutable, se mêle la fascination du sacré. L'homme est saisi devant la toute-puissance d'un ordre supérieur qui se manifeste par le gigantesque embrasement des éléments naturels: Sidérés, ils regardaient ce spectacle grandiose et terrifiant (p. 272); Longtemps, ils contemplèrent l'élément destructeur (p. 272); Le petit groupe s'était tu, saisi par la majesté terrifiante du spectacle. (p. 271)

Sur le plan des événements, la rupture avec l'ancienne vie est consommée dans un triple bénéfice pour l'avenir. Du côté profane, la forêt s'ouvre désormais aux projets de l'homme qui marquera le sol de son travail producteur:

[Eugène] se disait que [...] maintenant que la forêt avait disparu, il suffirait de nettoyer les débris et de labourer. Dans quelques années, presque toute sa ferme serait en culture. Et, comme on n'aurait plus à craindre le feu, ce serait mieux qu' avant. (p. 280)

Du côté sacré, le héros répond à l'appel d'une vocation supérieure à laquelle il sacrifie sa vie antérieure:

Quand j' ai vu venir cette muraille de flammes qui s' abattait sur nous, j'ai fait le vou solennel, si nous étions épargnés, de devenir prêtre et de consacrer ma vie aux missions les plus pauvres, aux âmes les plus délaissées. (p. 282)

Enfin, le feu sacrificiel conferre en même temps au héros une forme de jeunesse, gage de renouvellement de la vie:

Pour le moment, une pensée tournait dans [la] tête [de Rose] et elle s'y accrochait comme une noyée à une bouée : «L'enfant! " Advienne que pourra, il y aurait l'enfant... l'enfant d'Alex qu'elle portait dans son ventre. (p. 283)

Au terme de la pérégrination initiatique du héros dans la sombre forêt nordontarienne, la vie triomphe de la mort temporaire: un nouveau cycle commence et Hélène Brodeur l'a bien compris en poursuivant son récit dans une trilogie.

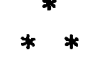

Le roman de Maurice de Goumois expose le parcours initiatique de François Duvalet, jeune employé de banque parisien qui, assoiffé de risque et d'action (p. 9), émigre au Canada pour tenter fortune. Il débarque à Montréal à l'automne 1928, à la veille de la crise économique et de la dépression. Décidé à réussir coûte que coûte, le jeune commis aux écritures accepte de s'engager comme bûcheron dans les chantiers de la North Star Lumber Company, situés au nord de Chapleau, petite localité perdue quelque part dans le nord-ouest de l'Ontario (p. 10). Rêvant d'accéder un jour à la direction d'une grande compagnie de bois (p. 25), l'émigré français se cogne aux dures réalités d'un pays 
totalement étranger. À ce citadin venu tout droit de Paris, image même de la civilisation, à ce modeste employé d'une administration routinière où il se sentait parfois comme un écureuil en cage (p. 9), le contact avec les contrées sauvages de la forêt du nord-ouest ontarien et la liberté d'une vie sans entraves réservent un choc aux retentissements considérables.

Dans ce second récit, la forêt est là aussi plus qu'une simple toile de fond exotique. Si celle-ci apparaît comme un lieu d'épreuves où le héros est contraint de pratiquer cinquante-six métiers et se trouve ainsi acculé à subir cinquante-six misères, elle représente, par ailleurs, l'épreuve suprême par laquelle François Duvalet refait peau neuve afin de s'implanter dans un nouveau pays, et quitte la défroque des vieux pays civilisés et le vernis de son urbanité pour découvrir non pas qui il est, mais ce qu'il est (p. 134). On assiste ainsi au périple initiatique qui valorise la découverte du «savoir magique», à la fois savoir pratique de la nature détenu par les hommes des bois et des métiers, connaissance abstraite comme celle de la langue anglaise, et, surtout, connaissance de soi. C'est, en somme, l'éducation totale de celui qui veut accéder à une nouvelle vie, celle d'un chef d'entreprise. La quête du «savoir magique» mise en scène dans ce roman est fréquente dans les contes populaires, l'enseignement constituant, on le sait, un trait caractéristique des rites d'initiation à travers le monde ${ }^{11}$.

De même qu'Alexandre Sellier dans le récit d'Hélène Brodeur, François Duvalet est soumis, dans un premier temps, à l'hostilité oppressante de la forêt. Dans ce monde de sapins et de durs à cuire, il est difficile pour un étranger parisien de trouver sa place. L'immensité du pays donne la mesure de la tâche: Personne ne lui avait parlé de la distance, mais deux jours et une nuit de chemin de fer ne lui laissèrent aucun doute: il courait vers l'autre bout du monde (p. 10-11). L'espace s'étend à perte de vue, échappe au contrôle du regard: la distance et encore de la distance! pensa Duvalet: ici tout est à l'autre bout du monde! (p. 72) Dans ce continent dispersé (p. 38), qui embrasse presque tout le septentrion (p. 37), la vastitudo s'amplifie du vide des lieux (p. 11, 37): l'homme qui marche ne rencontre âme qui vive (p. 36) car, dans ces régions aussi isolées, le premier voisin, quand il y en a un, est $a ̀ d e u x$ cent cinquante milles (p. 170). L'hiver, l'isolement s'accentue avec le silence prodigieux qui renvoie l'homme à sa solitude et au spectacle de la mort:

Seul au milieu de la nature ensevelie sur laquelle les sapins mornes et chargés semblaient pleurer en silence, Duvalet eut l'impression de profaner un immense sépulcre. Étreint d' un malaise indéfinissable, tout proche de la peur, il avança avec précaution. C'était terrible comme le grand silence pouvait être menaçant. (p. 73)

Car l'immensité grandiose du paysage est terrifiante. Elle opprime et aliène le néophyte. Les dangers y sont nombreux et inattendus. L'uniformité d'une nature anonyme et monotone qui offre toujours le même visage où que l'on tourne (p. 76) cache des ruses qui abolissent l'espace et le temps (p. 36). Par

11 Ibid., p. 132 et suivantes. 
exemple, l'homme inexpérimenté est condamné à errer pendant des heures jusqu'à l'épuisement complet, et les loups n'attendent que cela (p. 76). À la désorientation spatio-temporelle s'ajoute parfois l'égarement de l'esprit. Le silence, la solitude, la nuit, la faim et la fatigue perturbent, en effet, le sens des réalités :

Duvalet eut tout d'abord une hallucination lugubre. Pendant un moment, il crut qu'il perdait pied, qu'il s'enfonçait dans une masse qui n'avait plus rien de solide, mais refusait cependant de se refermer sur lui. (p. 73)

L'engourdissement dû au froid et au sommeil peuvent rallumer les illusions :

Accroupi contre un tronc, face à la flamme, il vient un moment où il ne se rendit plus compte qu'il avait cessé de songer. Il crut entendre une voix qui s'adressait à lui. (p. 74)

Sur la rivière, au sortir des rapides, l'élargissement du cours d'eau produit un effet magique qui semble arrêter le courant et auquel il est difficile d'échapper: le retour au silence [...] donnait l' illusion de rester immobile entre les rives en fuite (p. 162). Enfin, l'échec incessant du trappeur novice finit par tourmenter son imagination:

Autour de lui, il sentait la présence de tout un monde invisible. Dehors sur la neige, il rencontrait des pistes d'animaux partout; par une ironie cruelle, seules les siennes se rendaient aux pièges. Dans le grand silence de la nature endormie, il avait parfois l'impression que les bêtes se tapissaient à son approche pour mieux rire de lui. (p. 77)

Ainsi, dans ce pays nouveau, parmi une foule de choses qu'il n'a jamais vues, dont les aurores boréales (p. 19), et qu'il ne comprend pas, Duvalet demeure véritablement confondu: l'infini se mêlait au prosaïque pour fausser ce qu'il avait cru simple et normal (p. 20). À chaque tentative d'implantation dans ce pays étranger, l'univers impénétrable de la forêt montre un visage hostile qui risque de lui faire perdre pied.

La forêt ontarienne offre au jeune profane un spectacle peu engageant. Ici, c'est l'oppression d'une végétation noire et massive (p. 73); là, c'est la désolation d'un désert d'ipécéas et la dislocation du paysage sous les éboulis de rochers rebelles à la charrue (p. 37); plus au nord, c'est l'aspérité d'une végétation chétive et rare: la toundra parsemée de bruyères et arrosée de deltas boueux (p. 163, 164). La présence envahissante des bois domine les pauvres endroits isolés et perdus qui n'existent pour personne à l'extérieur sinon pour les archives des chemins de fer (p. 94) et dont les quelques masures éparpillées le long de la voie ferrée se fondent dans la végétation. La forêt se presse aussi, grise et sale, toutes formes confondues, autour des bourgades essaimées en des relais plus importants aux points stratégiques du réseau ferroviaire, comme à Chapleau (p. 38).

Dans ce pays inexploité et avare qui ne livre pas plus que ce qui peut s' emporter à dos d' homme ou entre les minces parois d' un canot (p. 162), la nature est indomptable. Le vrai maître est le climat (p. 37) et il est chiche dans ses 
belles saisons. L'hiver est long et, malgré la blancheur dont il s'entoure, présente un sombre tableau (p. 44). Le froid sec, un froid sibérien (p. 97), donne aux choses un relief impersonnel quasi hostile (p. 139). Sous son éclat, l'immobilité du paysage inerte et cristallisé est choquante: la neige au lieu de la recouvrir lui donn[e] une nudité accablante (p. 139). La surface des lacs emprisonnés par la glace devient inhospitalière (p. 73). Quant au printemps, il éclate bref et violent comme un fatal recommencement où tout est à refaire (p. 143). $\mathrm{Et}$, dans cette nature inculte, l'été surgit brusquement comme une débauche d'énergie, un gaspillage de ressources (p. 144). Sous la clarté oblique et sournoise de 1'automne qui enlève au paysage ses couleurs (p. 44), la silhouette découpée des conifêres se dresse dans l'air chargé d'effluves d' humus et de relents de résine [qui a] quelque chose d'opprimant (p. 13). Puis quand le soleil est bas à l'horizon, mais encore chaud, la myriade de bestioles insaisissables qui grouille dans cet air imbibé d'odeurs de débris organiques atteste, dit l'auteur, la domination hostile de la création (p. 35).

Pour ce qui est de la représentation de la mise à mort du candidat à l'initiation au «savoir magique», celle-ci prend une forme différente de l'initiation à la fonction sacerdotale du héros précédent, mais n'en est pas moins réelle. En effet, au lieu d'une mort instantanée dans une conflagration fulgurante, on assiste plutôt au dépouillement progressif d'une série de signes distinctifs qui définissent les grands traits de l'identité acquise de l'individu. Ce détachement graduel a pour but de mettre à nu le noyau dur de l'identité intime de la nature humaine: c'est l'initiation à la connaissance fondamentale de soi. Ainsi, en premier lieu, on assiste à la manifestation d'un «geste symbolique» qui ouvre le drame en rappelant la traversée du Styx à l'entrée des Enfers: Duvalet noie dans un lac son chapeau melon de Parisien, vestige d' un autre monde:

Il s'était débarrassé de ce qu'il avait acquis dans l'enceinte fortifiée $d^{\prime}$ un milieu conventionnel et inflexible. Maintenant, il se trouvait seul, hors de ses murs, sans autre point de repère que ses propres ressources. (p. 15)

À ce premier acte de répudiation du «vieil homme», vient se greffer une suite de faits qui mortifient l'amour-propre du héros. Ainsi, quand il arrive au camp de bûcherons, on lui soustrait son propre nom pour le surnommer «Frenchy», diminutif qui semblait désigner une race de second ordre (p. 14). D'autre part, on lui attribue des tâches indignes d'un vrai bûcheron (p. 16, 25); on l'humilie en le traitant de fille (p. 31) et on va jusqu'à lui retirer ses habits de travaillcur de la forêt avant de le renvoyer (p. 33). À l'hostilité de la forêt s'ajoute celle des hommes. Ces cuisantes expériences provoquent une première transformation profonde: personne n'eût reconnu en lui le citadin d'hier. Son nouveau milieu l'avait déjà absorbé. Comme une mauvaise herbe dans un champ inculte, il n'y faisait plus tache (p. 35). La transformation est brutale: il se débarrasse d'une foule de notions (p. 61) pour acquérir le rythme de la grande nature: la distance faisait maintenant partie du pays et marcher devint une habitude (p. 107). La domination de la forêt s'étend ainsi peu à peu à ses manières: [s] a cabane finit par ressembler à une tanière (p. 76). 
Au bout de cinq mois de ce régime nouveau, il eût été difficile, remarque l'auteur, de le reconnaître: en apparence, le pays l'avait absorbé (p. 130). Mais le véritable détachement, la transformation la plus profonde qui fera sauter tout à fait l'écorce de l'homme civilisé viendra quand François Duvalet devra affronter non plus les embûches extérieures ou les affronts personnels, mais sa vraie nature humaine. L'occasion qui se présente constitue alors l'étape ultime de la mise à mort figurée du néophyte; il atteint dès lors le paroxysme de l'épreuve du «savoir magique» où il découvre en même temps qu'il la montre son absorption totale par le monde de la forêt, force aveugle et violente. Ainsi, devant le saccage de son camp par un faux rival, François Duvalet ressent une impulsion incontrôlable (p. 160), d'une violence sauvage qui appelle à la vengeance et le pousse au bord du crime pour défendre une cabane délabrée chèrement acquise. C'est la mise à nu de ses instincts les plus profonds, noyau dur de la condition humaine. Si le héros a pu résister à l'élan meurtrier, c'est encore, dit l'auteur, grâce à la force infaillible de la nature.

\section{Mais la puissance qui était intervenue et l'avait empêché de devenir meurtrier ne provenait pas de lui seul. Une volonté plus forte que la sienne, qui devait être la même que celle qui fait les saisons, avait retenu son bras. Ce fut la seule explication qu'il put se donner. Donc, il n'était pas si seul. (p. 146)}

On surprend ici le saisissement de l'homme devant l'étendue de la manifestation d'une toute-puissance supérieure en même temps que l'acquisition du bienfait d'un enseignement, dernier stade de l'initiation:

Ne regarder que soi empêche de voir autour de soi, finit-il par se dire; c'est le genre d'aveuglement qui mène fatalement à la turpitude! Plus soumis, il se sentit plus en paix. Rien ne servait de forcer la main à son destin; il vivrait au jour le jour au lieu de toujours interroger l'avenir.

Il n'avait qu'à observer la nature; malgré un désordre apparent, malgré la profusion, rien ne s'y produisait avant le temps. (p. 146)

Au sortir de la forêt qu'il finit par quitter (comme Alexandre Sellier), le nouvel initié est muni d'un bagage de connaissances qui lui assure un travail inespéré dans son nouveau pays d'adoption et lui ouvre ainsi le chemin d'une vie nouvelle: Qui sait, conclut Duvalet, mon aventure ne fait peut-être que commencer (p. 263). Ainsi va le cycle de la vie.

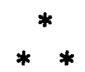

Un bref regard rétrospectif sur la lecture proposée dans cette analyse de deux romans ontarois inspirés de faits historiques et de faits biographiques, montre, en premier lieu, que derrière ces deux romanciers se cache un conteur et que derrière le conteur surgit inévitablement la forêt. Une deuxième observation concerne, cette fois, la présence imposante des bois qui innerve tout le récit et lui donne vie: plus qu'un simple rideau de scène ou encore qu'un lieu privilégié d'action, la forêt constitue le ressort essentiel du drame sans lequel il 
ne pourrait y avoir d'action. Enfin, une troisième constatation renvoie à l'hostilité apparente de la forêt qui décrit moins une réalité géographique qu'elle ne dévoile les traits fondamentaux de son efficacité fonctionnelle dans le récit. En effet, considérée comme l'entrée du royaume des morts, la forêt doit revêtir des propriétés telles qu'il est permis de penser que plus elle est éloignée du monde, plus elle se rapproche de cet autre monde où la mort, en son sein, trame à son aise le fil de la vie.

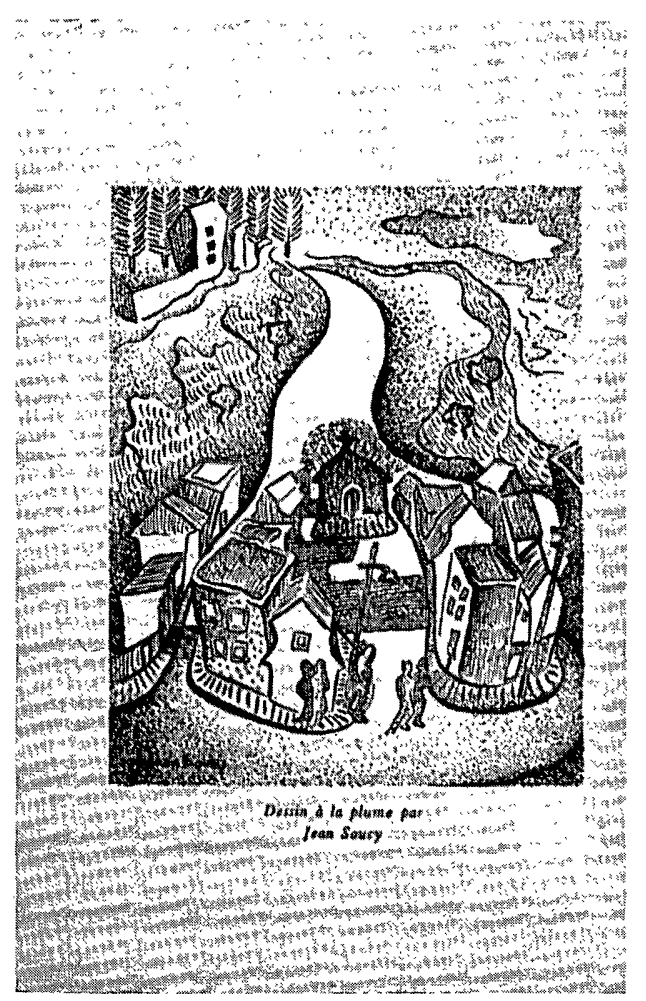

